\title{
REVISÃO TEÓRICA INTEGRATIVA ACERCA DOS POSSÍVEIS FATORES QUE LEVAM A TENTATIVA DE SUICÍDIO
}

\section{REVISÃO INTEGRATIVA}

LEÃO, Marco Härter ${ }^{1}$

DUARTE, Luiz Felipe Bastos ${ }^{2}$

LEÃO, Marco Härter. DUARTE, Luiz Felipe Bastos. Revisão teórica integrativa acerca dos possíveis fatores que levam a tentativa de suicídio. Revista Científica Multidisciplinar Núcleo do Conhecimento. Ano 05, Ed. 11, Vol. 04, pp. 101-110. Novembro de 2020. ISSN: 2448-0959, Link de acesso:https://www.nucleodoconhecimento.com.br/psicologia/tentativa-de-suicidio

\section{RESUMO}

Existem múltiplos fatores relacionados à tentativa de suicídio que, na maioria dos casos, estão vinculada à vontade de romper com uma realidade de grande sofrimento psíquico. Esse estudo tem como objetivo realizar uma revisão integrativa de literatura, em artigos de língua portuguesa dos últimos 5 anos acerca dos possíveis fatores de risco que levam a tentativa de suicídio. Foram utilizados para a busca os seguintes descritores: suicídio, fatores, risco e tentativa. Sendo selecionados a partir de uma triagem 10 artigos relacionados ao asssunto do trabalho e separados posteriormente em 5 categorias para análise: fatores de risco na infância, fatores de risco na adolescência, fatores de risco na adultez, fatores de risco na velhice e fatores de risco gerais predisponentes. Os resultados apontam as principais causas de suícidio em cada faixa etária: conflitos familiares na infância; construção da identidade na

\footnotetext{
${ }^{1}$ Acadêmico de Psicologia.

2 Orientador. Graduado em Psicologia; Mestre em Ciências da Comunicação. 
adolescência; relacionamentos interpessoais na adultez; e falta de autonomia na velhice.

Palavras-chave: fatores de risco, comportamento, suicídio.

\section{INTRODUÇÃO}

O suicídio possui múltiplos fatores, que podem variar conforme cada indivíduo, alguns mais evidentes em alguma faixa etária e, outros, comuns a todas as idades. É necessário o conhecimento dos possíveis fatores que levam a tentativa suicídio, destarte, o objetivo do presente trabalho é realizar uma revisão integrativa de literatura a respeito do tema e descobrir os possíveis fatores que levam a tentativa de suicídio, para, assim, auxiliar aqueles que lidarão com isto e para que se possa tentar compreender as causas de novas tentativas e evitar a consumação do ato, que pode levar ao fim de uma vida.

Segundo Botega (2014), o suicídio é uma das principais causas de mortes de pessoas entre 15 e 44 anos de idade. A maioria dos casos envolvem algum transtorno mental, sendo as principais causas depressão, transtorno de humor bipolar, ou álcool e outras substâncias psicoativas.

Para o Conselho Federal de Psicologia (2013), os fatores que envolvem o suicídio são múltiplos (biopsicossocial), sendo os principais deles: relacionados a transtornos mentais, depressão, ou abuso de substâncias psicoativas, o que representa $90 \%$ dos casos. Todo caso de suicídio é um marcador de sofrimento psíquico, ou transtorno mental. O sofrimento psíquico, é algo existencial, que todos nós experimentamos em menor ou maior intensidade durante a vida, mas associado a um transtorno psiquiátrico, em casos graves, pode oferecer um maior risco de suicídio.

Para Owens; Horrocks e House (2002), uma tentativa de suicídio aumenta consideravelmente o risco de sua futura concretização em relação aos índices populacionais em geral. Segundo Hillman (1993), o suicídio não é uma síndrome, nem sintoma, ele é algo complexo, ainda mais que uma psicose, tensão sexual, ou 
violência física, pois não é um problema de vida, mas um problema de vida e morte. O indivíduo não simplesmente comete o suicídio, mas antes preenche certas condições para que cometa a tentativa, essas que teriam a ver com a sociedade em que vive e seu mundo interno. Seria necessário aproximar o indivíduo de seu grupo de convívio, pois o isolamento, causado por problemas vividos como divórcio, fracasso, viuvez, etc, é uma influência desintegradora e, frente a isto o ser humano pode tentar se suicidar. Este autor destaca também os aspectos inconscientes do indivíduo, para ele antes de nos aproximarmos do problema do suicídio devemos primeiramente compreender a vida desse. É necessário conhecer os aspectos conscientes e inconscientes da personalidade do indivíduo, suas indagações internas tais como sonhos, fantasias e modo de apercepção para assim se formar um quadro adequado dos motivos que levam ao ato suicida.

\section{METODOLOGIA}

O presente estudo constitui-se numa revisão integrativa de literatura. Para tal, foi realizada uma busca nas seguintes bases de dados online: Scielo, PEPSIC e Google Acadêmico.

Os descritores utilizados foram "suicídio" em combinação com outros, tais como: fatores, risco e tentativa. Foram adotados critérios de inclusão: artigos em português, com o tema fatores de risco que levam a tentativa de suicídio, com ano de publicação entre 2013 e 2018. Enquanto os critérios de exclusão, foram: artigos repetidos, artigos que fugissem da temática, artigos em língua inglesa e com data de publicação anteriores a 2013. Após realizada uma leitura dos artigos encontrados, procedeu-se uma organização dos dados a partir das seguintes categorias: fatores de risco na infância, fatores de risco na adolescência, fatores de risco na adultez, fatores de risco na velhice e fatores de risco gerais predisponentes de forma a categorizar as informações consideradas importantes para o objetivo geral deste trabalho. 


\section{APRESENTAÇÃO DE RESULTADOS}

Após a realização da pesquisa segundo os descritores propostos foram encontrados os seguintes resultados: 9 artigos na base PEPSIC, 25 no Scielo e 35 resultados no Google Acadêmico.

Após a leitura dos 69 artigos encontrados foram excluídos 59 artigos por fugirem do tema desta pesquisa, ou por serem repetidos. Desta forma foram considerados, para fins de estudo, 10 artigos relevantes, que seguem abaixo identificados por nome do autor, título, objetivo geral e metodologia (Quadro 1).

Quadro 1 - Características Dos Artigos Selecionados Nas Bases De Dados Digitais $(\mathrm{N}=10)$.

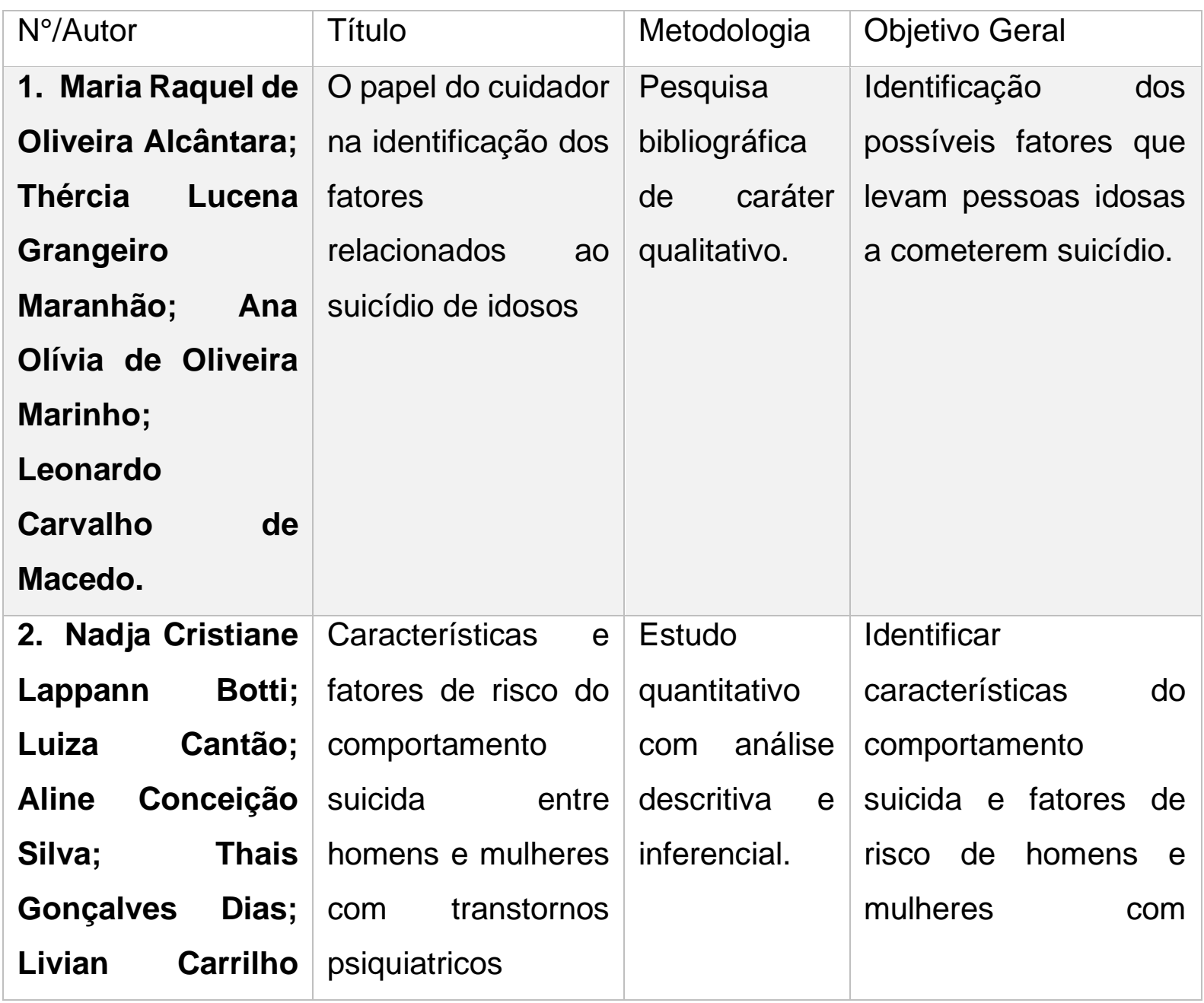




Menezes; Ramon
Azevedo Silva de
Castro.

3. Ana Carolina Rodrigues

Pereira; Vanessa

Cristina

Alvarenga.

4. Luiza de Lim

Braga; Débora

Dalbosco

Dell'Aglio.

5.

Benincasa;

Manuel Morgado Rezende.

6.

Yohanna

Cerqueira; Patrícia Lima. transtornos
psiquiátricos.

Compreender de literatura. suicida. sobre os fatores de sistemática comportamento risco

Miria Tristeza e suicídio entre adolescentes: fatores de risco e proteção

Suicídio: a prática Revisão do psicólogo e os sistemática principais fatores de de literatura. risco e proteção na Revisão sistemática de literatura. depressão e gênero
Discutir fatores de risco de suicídio na adolescência sob uma perspectiva desenvolvimental.

Grupo focal Identificar os fatores para coleta e de risco e proteção análise de relacionados ao dados. suicídio.

Trazer uma contribuição ao campo da psicologia e áreas afins a respeito do comportamento suicida.

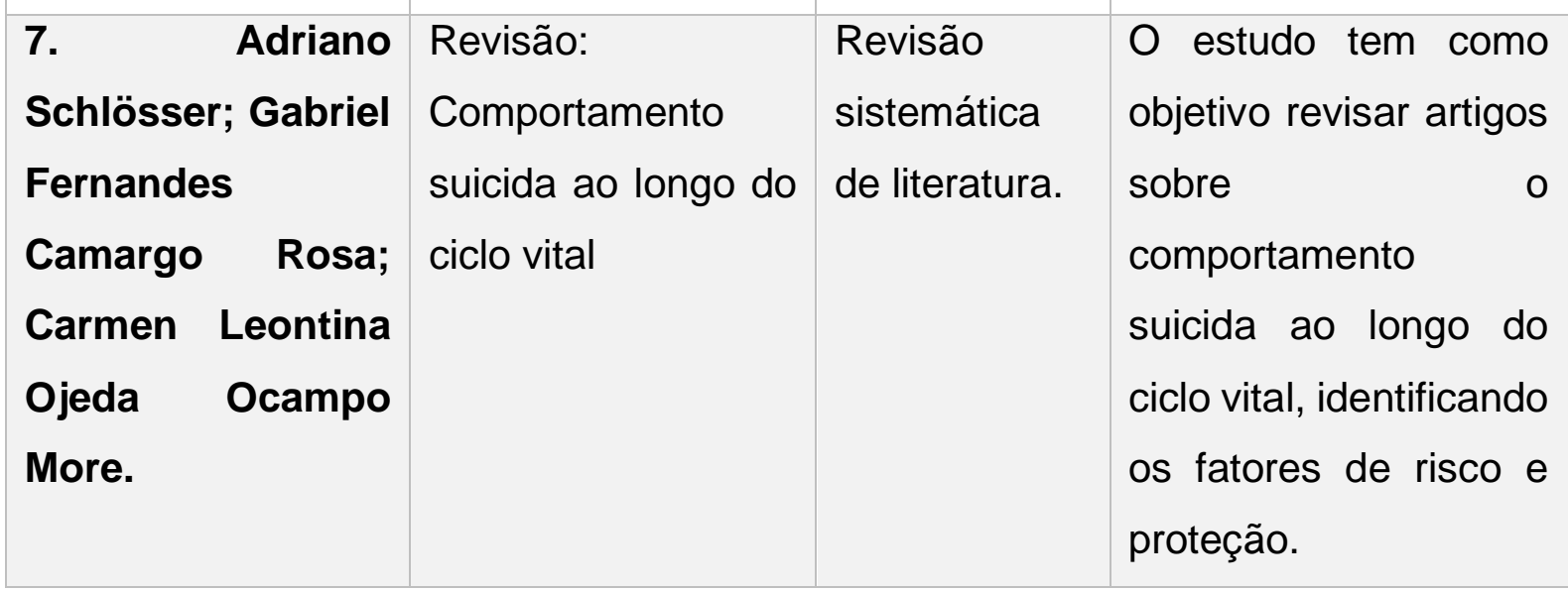




\begin{tabular}{|c|c|c|c|}
\hline $\begin{array}{l}\text { 8. Marta Brás; } \\
\text { Saul Jesus; } \\
\text { Claúdia Carmo. }\end{array}$ & $\begin{array}{l}\text { Fatores } \\
\text { psicológicos de } \\
\text { risco e protetores } \\
\text { associados à } \\
\text { ideação suicida em } \\
\text { adolescentes }\end{array}$ & $\begin{array}{lr}\text { Análise } & \text { e } \\
\text { coleta } & \text { de } \\
\text { dados } & \text { de } \\
\text { grupo } & \\
\text { amostral. } & \end{array}$ & $\begin{array}{l}\text { Estudar a relação dos } \\
\text { fatores psicológicos de } \\
\text { risco e proteção com a } \\
\text { ideação suicida. }\end{array}$ \\
\hline $\begin{array}{l}\text { 9. Girliani Silva } \\
\text { Sousa; Marilia } \\
\text { Suzi Pereira dos } \\
\text { Santos; Amanda } \\
\text { Tabosa Pereira da } \\
\text { Silva; Jaqueline } \\
\text { Galdino } \\
\text { Albuquerque } \\
\text { Perrelli; Evertgon } \\
\text { Botelho Sougey. }\end{array}$ & $\begin{array}{l}\text { Revisão de } \\
\text { literatura sobre } \\
\text { suicídio na infância }\end{array}$ & $\begin{array}{l}\text { Revisão } \\
\text { sistemática } \\
\text { de literatura. }\end{array}$ & $\begin{array}{l}\text { Analisar a literatura } \\
\text { específica sobre os } \\
\text { fatores associados ao } \\
\text { comportamento } \\
\text { suicida em crianças } \\
\text { com até } 14 \text { anos. }\end{array}$ \\
\hline $\begin{array}{l}\text { 10. Maria Cecília } \\
\text { de Souza Minayo; } \\
\text { Fátima Gonçalves } \\
\text { Cavalcante. }\end{array}$ & $\begin{array}{l}\text { Suicídio entre } \\
\text { pessoas idosas: } \\
\text { revisão de literatura }\end{array}$ & $\begin{array}{l}\text { Revisão } \\
\text { sistemática } \\
\text { de literatura. }\end{array}$ & $\begin{array}{l}\text { Promover uma revisão } \\
\text { de literatura a cerca do } \\
\text { tema suicídio entre } \\
\text { pessoas idosas. }\end{array}$ \\
\hline
\end{tabular}

Fonte: O Autor (2018).

\section{DISCUSSÃO}

O presente estudo objetivou realizar uma revisão integrativa de literatura, em artigos de língua portuguesa dos últimos 5 anos acerca dos possíveis fatores de risco que levam a tentativa de suicídio. A partir de uma leitura prévia dos artigos encontrados (Quadro 1) criou-se uma divisão categórica dos assuntos relevantes ao objetivo desse trabalho. As categorias a que se chegou são: fatores de risco na infância, fatores de risco na adolescência, fatores de risco na adultez, fatores de risco na velhice e fatores 
gerais predisponentes da tentativa de suicídio. Sendo evidenciado em cada uma delas os fatores de risco que levam a tentativa de suicídio.

Antes da elucidação das categorias faz-se necessário conceituar dois termos importantes, presentes na leitura delas, que são: tentativa de suicídio e ideação suicida. Segundo Ferreira (2008), conceitua-se uma tentativa de suicídio qualquer ato não fatal, autoinfligido, que atente contra a própria vida. Enquanto, a ideação suicida, segundo Silva (2006), constitui-se na idealização e planejamento da morte de si mesmo, ou seja, é a fantasia, ou desejo que precede a tentativa de suicídio.

A primeira categoria "fatores de risco na infância" aborda através dos artigos 7 e 9 as principais causas das tentativas de suicídio da faixa etária de 5 e 14 anos. Os conflitos familiares são um grande indicador, crianças que participam de um ambiente familiar hostil, com falta de diálogo e normas rígidas estão mais propensas a desenvolver ideações suicidas. Assim também, a presença de parentes depressivos, ou pais que tentaram o suicídio também contribui para aumentar o risco de ideações suicidas nessa faixa etária. Outros fatores estressores como divórcio dos pais, ou histórico de abuso sexual, estão associados também aos fatores de risco. Fatores psíquicos, cognitivos e abuso de substâncias fazem parte das causas de suicídio, sendo o baixo desempenho escolar um grande predisponente das tentativas de suicídio, este que pode estar diretamente ligado à algum tipo de problema cognitivo como TDAH (transtorno de Déficit de Atenção e Hiperatividade). A criança no meio escolar está sob risco de sofrer bullying, um tipo de agressão física, ou psíquica com fim de humilhá-la, que pode causar seu isolamento e morte. Além disso o baixo rendimento escolar pode causar-Ihe medo das críticas e pressões sociais e familiares. Qualquer mudança no comportamento da criança pode ser um fator de risco, visto que as mesmas costumam se isolar e agir de forma estranha nas semanas que antecedem seu suicídio, evitando até mesmo ir à escola no dia da tentativa. É importante atentar aos traços de personalidade da criança que podem estar ligados a fatores de risco, visto que crianças mais raivosas, ansiosas e impulsivas, ou com traços depressivos apresentam maiores chances da tentativa. 
A segunda categoria "fatores de risco na adolescência" aborda através dos artigos 4, 5, 7 e 9 as principais causas de suicídio, na faixa etária de 15 a 19 anos de idade. A própria adolescência como fase de vida é um fator de risco, visto que se trata de um momento de crise e de construção da identidade (personalidade), com baixa tolerância à frustração, e vulnerabilidade do self. A tristeza, a falta de motivação, o sentimento de desesperança, e sintomas depressivos, são fatores de risco que podem levar à tentativa de suicídio, visto que jovens que apresentam tais quadros estão mais predispostos a serem vítimas de ideações suicidas. Esses fatores, por sua vez, podem estar ligados à problemas familiares, relacionamentos amorosos, traição de amigos, sentimentos de desproteção, questões financeiras, ou à falta de privacidade e escuta. Alguns fatores que ocorrem na adolescência assemelham-se as causas do suicídio infantil, como problemas cognitivos, psíquicos, dificuldades escolares, abuso de substâncias químicas e bullying, sendo os maiores predisponentes da ideação suicida.

A terceira categoria "fatores de risco na adultez" explora através dos artigos 2, 6 e 7 as principais causas de suicídio, na faixa etária de 35 a 54 anos. O maior afetado nessa fase é o homem, que devido à culturalidade patriarcal do "homem provedor", ser mais vulnerável quando se encontra em situações de desemprego, ou empobrecimento. Além disso, nessa fase, independente do genêro, faz-se importante a criação de vínculos, e a ausência de isolamento, visto que adultos sem parentes próximos, amigos, ou algum tipo de relacionamento afetivo/amoroso, estão mais sucetíveis às ideações suicidas. A perda de relacionamentos interpessoais, também pode estar relacionada à ideação suicida demonstrando que a perda de vínculos pode ser tão grave quanto a sua ausência. Por fim, um histórico familiar de doenças psiquiátricas, principalmente depressão, também, pode estar associados a um maior risco de suicídio, além disso, como em outras faixas etárias o adulto também sofre abuso de substâncias químicas, que podem precipitar ou predispor, a tentativa de suicídio.

A quarta categoria "fatores de risco na velhice" composta pelos artigos 1, 3, 6, 7 e 10 exploram a faixa etária acima dos 75 anos de idade, na qual o idoso encontra-se mais vulnerável à tentativa de suicídio. Os principais fatores de risco encontrados 
neste grupo são a perda de autonomia e falta de satisfação das necessidades básicas, que podem estar relacionados à doenças físicas, ou psíquicas e à falta de cuidado da família, ou cuidador. Nessa fase da vida acontece um grande número de transformações biológicas, sociais e psíquicas, que afetam autoestima, os predispondo a tentativa de suicídio, afim de aliviar os sentimentos de angústia, desesperança e sofrimento. Sendo que os pacientes deprimidos, ou depressivos apresentam até duas vezes mais risco de tentativa de suicídio. Por outro lado, questões como aposentadoria, perda da função social, ou morte de pessoas próximas estão também relacionados ao risco de comportamento suicida nessa idade. Outros fatores contribuintes podem ser o ambiente hostil e a falta de afetividade familiar, já que o idoso se vê fragilizado devido às limitações da idade.

\section{A quinta e última categoria, "fatores gerais predisponentes da tentativa de} suicídio", apresenta informações encontradas nos 10 artigos analisados, e discorre os fatores de risco da tentativa de suicídio em todas as faixas etárias. Os principais fatores de risco da ideação suicida estão relacionados a transtornos mentais e ao abuso de substâncias químicas (lícitas e ilícitas). Também existem especificidades de gênero: enquanto nos homens há maior risco de suicídio devido ao uso de drogas, nas mulheres têm-se como maior fator de risco um histórico de abusos de todo tipo, bem como, o transtorno de humor. Por fim, diversos outros fatores podem ser considerados predisponentes da tentativa de suicídio independente da faixa etária: histórico de suicídio na família; tentativas anteriores; doenças físicas e psicológicas incapacitantes; isolamento social e sentimento de solidão; impotência e desesperança.

São citados na cartilha brasileira de prevenção ao suicídio, segundo Unimed (2018), fatores de risco ao suicídio que vão de encontro as informações dos artigos da última categoria. Dentre os principais fatores estão problemas socioculturais, genéticos, filosóficos existenciais e ambientais. Sendo agravantes conflitos, desastres, violência, abusos, ou perdas, isolamento social e transtornos mentais.

Segundo a Organização Mundial da Saúde (2000), aqueles que tentaram o suicídio na maioria dos casos possuem algum transtorno mental diagnosticável. Os grupos 
diagnósticos, em ordem de risco são: depressão (todas as formas); transtorno de personalidade (antissocial e boderline com traços de impulsividade, agressividade e frequentes alterações de humor); alcoolismo (e/ou abuso de substância em adolescentes); esquizofrenia; transtorno mental orgânico. Portanto, embora, hajam diversos fatores de risco, envolvidos numa tentativa de suicídio, evidencia-se que a presença de um transtorno mental é a principal causa independente da faixa etária.

\section{CONSIDERAÇÕES FINAIS}

Levando em conta o objetivo geral do presente estudo, que é realizar uma revisão integrativa de literatura acerca dos possíveis fatores que levam a tentativa de suicídio, as literaturas indicam que são múltiplos os fatores determinantes da tentativa de suicídio. São fatores gerais, comuns a todas as faixas etárias, o isolamento social, o abuso de substâncias químicas, a falta de afeto e os problemas psicológicos. São fatores pontuais, relacionados às principais causas de suicídio, em cada faixa etária: conflitos familiares na infância; construção da identidade na adolescência; relacionamentos interpessoais na adultez; e falta de autonomia na velhice.

Em todos os casos de tentativa de suicídio, o que se pode notar é um rompimento, ou uma tentativa de romper, com a realidade atual, que pode acontecer de forma consciente, ou inconsciente. Nos casos de doenças mentais e abuso de substâncias químicas, devido ao rompimento com a realidade, ou perda das capacidades plenas mentais, o indivíduo torna-se vulnerável à tentativa de suicídio, pois não se encontra completamente ciente dos seus atos. A alteração do estado mental interfere na forma como ele encara a realidade, pois está fragilizado devido à percepção alterada do mundo ao seu redor. Já quando o ser humano não encontra mais significado em sua existência, sente-se solitário, enfrenta um grande número de mudanças biopsicossociais, dores, ou angústias o suicídio torna-se uma alternativa para romper com sua realidade atual e o sofrimento proporcionado por ela.

O presente estudo encontrou algumas dificuldades, tais como a limitação de literatura devido a restrição do estudo a artigos de língua portuguesa. Além disso, utilizou-se apenas um recorte das ideias originais dos autores dos artigos, sendo pouco os 
conteúdos acerta dos fatores de risco que podem levar a tentativa de suicídio, encontrados dentro dessa delimitação. Cabe aos profissionais da área saúde e futuros estudos, explorar melhor os fatores de risco que podem levar a tentativa de suicídio.

\section{REFERÊNCIAS}

Alcântara, Maria Raquel de Oliveira; Maranhão, Thércia Lucena Grangeiro; Marinho, Ana Olivia de Oliveira; Macedo, Leonardo Carvalho (2018). O papel do cuidador na identificação dos fatores relacionados ao suicídio de idosos. Revista multidisciplinar de de psicologia. Recuperado através de: < https://idonline.emnuvens.com.br/id/article/view/1021>

Benincasa, Miria (2013). Tristeza e suicídio entre adolescentes: fatores de risco e proteção. Boletim de psicologia. Recuperado através de: < http://pepsic.bvsalud.org/pdf/bolpsi/v56n124/v56n124a07.pdf>

Botega, José Neury (2014). Comportamento suicida: epidemiologia. Campinas, São Paulo. Recuperado através de: < http://www.scielo.br/pdf/pusp/v25n3/0103-6564pusp-25-03-0231.pdf>

Botti, Nadja Cristine Lappan; Cantão, Luiza; Silva, Aline Conceição; et al (2018). Características e fatores de risco do comportamento suicida entre homens e mulheres com transtornos psiquiátricos. Cogitare enfermagem. Recuperado através de: <http://docs.bvsalud.org/biblioref/2018/05/883500/54280-231505-1-pb.pdf>

Braga, Luiza de Lima; Dell'Aglio, Débora Dalbosco (2013). Suicídio na adolescência: fatores de risco, depressão e gênero. Universidade Federal do Rio Grande do Sul. Recuperado através de: http://revistas.unisinos.br/index.php/contextosclinicos/article/viewFile/ctc.2013.61.01/ 1533>

Carmo, Claúdia; Jesus, Saul; Brás, Marta (2016). Fatores psicológicos de risco e protetores associados à ideação suicida em adolescentes. Psicologia, Saúde e 
Doenças.

Recuperado

através

de:

http://www.scielo.mec.pt/pdf/psd/v17n2/v17n2a03.pdf>

Cerqueira, Yahanna Shneideider; Lima, Patrícia Valle de Albuquerque (2015). Suicídio: a prática do psicólogo e os principais fatores de risco e proteção. Revista IGT na Rede. Recuperado através de: < http://pepsic.bvsalud.org/pdf/igt/v12n23/v12n23a10.pdf>

Conselho Federal de Psicologia (2013). Suicídio e os desafios para psicologia. Brasília. Pág. 10-19. Recuperado através de: < https://site.cfp.org.br/wpcontent/uploads/2013/12/Suicidio-FINAL-revisao61.pdf>

De Sousa, Girlani Silva; Dos Santos, Marília Suzi Pereira; Da Silva, Amanda Tabosa Pereira; et al (2017). Revisão de Literatura sobre suicídio na infância. Universidade Federal de Pernambuco. Recuperado através de: http://www.scielo.br/pdf/csc/v22n9/1413-8123-csc-22-09-3099.pdf

Ferreira, Renato (2008). O suicídio. Universidade de Coimbra. Recuperado através de: http://www4.fe.uc.pt/fontes/trabalhos/2008025.pdf

Fontes, Martins (2000). Émile Durkheim: O suicídio. São Paulo. Pág.31-50. Recuperado através de:

https://edisciplinas.usp.br/pluginfile.php/3952040/mod_resource/content/1/2000_Dur kheim_0\%20Suicidio\%20-\%20livro\%20inteiro.pdf>

Fukumitsu, Karina Okajima (2014). O psicoterapeuta diante do comportamento suicida. Universidade de São Paulo, SP, Brasil. Recuperado através de: < http://www.scielo.br/pdf/pusp/v25n3/0103-6564-pusp-25-03-0270.pdf>

Hesketh, José Luiz; Castro, Archimedes Guimarães (1978). Fatores correlacionados com a tentativa de suicídio. Revista de saúde pública, São Paulo. Recuperado através de: < http://www.scielo.br/pdf/rsp/v12n2/05.pdf> 
Hillman, James (1993). Suicídio e Alma. Petrópolis. Pág. 69-127. Recuperado através de: < https://www.passeidireto.com/arquivo/19070743/suicidio-e-alma---hillman>

Kuckzynski, Evelyn (2014). Suicídio na infância e adolescência. Hospital das clínicas da faculdade de medicina da universidade de São Paulo. Recuperado através de: < http://www.scielo.br/pdf/pusp/v25n3/0103-6564-pusp-25-03-0246.pdf>

Minayo, Maria Cecília (2010). Suicídio entre pessoas idosas: revisão da literatura. Revista de Saúde Pública, Rio de Janeiro. Recuperado através de: < http://www.scielo.br/pdf/rsp/v44n4/20.pdf>

Organização Mundial da Saúde (2000). Prevenção do suicídio: um manual para profissionais da saúde em atenção primária. Genebra. Pág. 5. Recuperado através de:

https://www.who.int/mental_health/prevention/suicide/en/suicideprev_phc_port.pdf

Owens, D., Horrocks, J., \& House, A. (2002). Tentativa de suicídio fatal e não fatal. Revisão sistemática. British Jornal de Psiquiatria. Pág. 181. Recuperado através de: $<\quad$ https://www.cambridge.org/core/services/aop-cambridgecore/content/view/721FD68B3030C46E2070CC08CA869523/S000712500002715Xa .pdf/fatal_and_nonfatal_repetition_of_selfharm.pdf>

Pereira, Ana Carolina Rodrigues; Alvarenga, Vanessa Cristina (2016). Suicídio: um estudo sobre os fatores de risco. Revista educação, saúde e meio ambiente. Recuperado através de: < https://docplayer.com.br/82483716-Suicidio-um-estudosobre-os-fatores-de-risco-ana-carolina-rodrigues-pereira-5-vanessa-cristinaalvarenga-6.html>

Schlosser, Adriano; Rosa, Gabriel Fernandes Camargo; More, Carmen Leontina Ojeda Ocampo (2014). Revisão: comportamento suicida ao longo do ciclo vital. Temas em Psicologia. Recuperado através de: http://pepsic.bvsalud.org/pdf/tp/v22n1/v22n1a11.pdf> 
Silva, Viviane Franco (2006). Ideação suicida: um estudo de caso-controle na comunidade. UNICAMP, Universidade de Campinas. Recuperado através de: < http://repositorio.unicamp.br/bitstream/REPOSIP/311457/1/Silva_VivianeFrancoda M.pdf>

Unimed (2018). Cartilha de Prevenção ao Suicídio. Recuperado através de: < http://unimed.coop.br/portalunimed/cartilhas/prevencao-ao-suicidio/cartilha.pdf>

Enviado: Outubro, 2020.

Aprovado: Novembro, 2020. 